\title{
NOVAS COMBINAÇÕES, NOVAS OCORRÊNCIAS E NOTAS SOBRE ESPÉCIES POUCO CONHECIDAS, PARA AS ORQUÍDEAS DO BRASIL
}

Recebido em 10.02.92. Aceito em 23.03.92

Fábio de Barros ${ }^{1}$

RESUMO - (Novas combinações, novas ocorrências e notas sobre espécies pouco conhecidas, para as orquídeas do Brasil). Duas novas combinações são propostas para orquídeas brasileiras: Cyrtopodium polyphyllum (Vell.) Pabst ex F. Barros e Pseudolaelia canaanensis (Ruschi) F. Barros. Maxillaria perparva Garay \& Dunsterv. é citada pela primeira vez para o Brasil. São apresentadas ilustrações de Maxillaria friedrichsthalii Rchb. f. e Platystele ovalifolia (Focke) Garay \& Dunsterv., duas espécies coletadas raramente no Brasil.

Palavras-chave: Orchidaceae, Cyrtopodium, Maxillaria, Plastytele, Pseudolaelia.

\begin{abstract}
New combinations, new occurences and notes about species bad known for the orchids of Brazil). Two new combinations are proposed for Brazilian orchids: Cyrtopodium polyphyllum (Vell.) Pabst ex F. Barros and Pseudolaelia canaanensis (Ruschi) F. Barros. Maxillaria perparva Garay \& Dunsterv. is reported for the first time for Brazil. Illustration of Maxillaria friedrichsthalii Rchb. f. and Platystele ovalifolia (Focke) Garay \& Dunsterv., two species seldom collected in Brazil, are given.
\end{abstract}

Key words: Orchidaceae, Cyrtopodium, Maxillaria, Plastytele, Pseudolaelia.

\section{Introdução}

Com o presente trabalho, inicia-se uma série em que se pretende abordar, conjuntamente, problemas taxonômicos e fitogeográficos relativos às orquídeas brasileiras, cujo volume não justifique a publicação de trabalhos individuais.

Neste primeiro trabalho de série são propostas duas novas combinações: Cyrtopodium polyphyllum (Vell.) Pabst ex F. Barros e Pseudolaelia canaanensis (Ruschi) F. Barros; a primeira corresponde ao nome que deve ser adotado para espécie formalmente conhecida como Cyrtopodium paranaense Schltr., a segunda é baseada em Renata canaanensis Ruschi. Além disso, Maxillaria perparva Garay \& Dunsterv.

\footnotetext{
' Instituto de Botânica, Caixa Postal 4005, 01061-970, São Paulo-SP, Brasil.
} 
é citada pela primeira vez para o Brasil e são apresentadas ilustrações completas de Maxillaria friedrichsthalii Rchb. f. e Plastytele ovalifolia (Focke) Garay \& Dunsterv.

\section{Resultados e discussão}

Cyrtopodium polyphyllum (Vell.) Pabst ex F. Barros comb. nov. Epidendrum polyphyllum Vell., Fl. Flum. Ic. 9: t.17. 1827.

Cyrtopodium paranaense Schltr., Fedde repert. 16:333. 1920 syn. nov.

Tradicionalmente Epidendrum polyphyllum Vell. tem sido tratado como sinônimo de Cyrtopodium andersonii R. Br. por vários autores (Cogniaux, 1898; Hoehne, 1942, 1952; Pabst \& Dungs, 1975). A ilustração da espécie apresentada por Vellozo (1827) apesar de não ser perfeita, deixa perceber que as flores possuem labelo com lobos laterais estreitos e encurvados e lobo central destacado dos laterais, além de sépalas lanceoladas. Essas características não concordam com as da espécie brasileira que vem sendo tratada com o nome de Cyrtopodium andersonii. Essas mesmas características, aliadas à distribuição geográfica e à cor das flores indicada por Vellozo (1881) mostram que se trata de outa espécie, conhecida até o momento, por Cyrtopodium paranaense Schltr. Esse fato já havia sido percebido por Pabst que, embora não tenha publicado a nova combinação, deixou-a indicada em algumas etiquetas de herbário.

É importante ressaltar, por outro lado, que a verdadeira Cyrtopodium andersonii é endêmica das Indias Ocidentais (Garay \& Sweet 1974) não ocorrendo no Brasil; a espécie brasileira comumente tratada por esse nome deveria receber o nome de Cyrtopodium flavum Link \& Otto ex Rchb. f.

Material estudado: Brasil: Paraíba: João Pessoa, B. Pickel, 16-XI-1933 (SP). Paraná: Paranaguá, Sítio do Meio, G. Hatschbach 2658, 28-IX-1951 (SP). Pernambuco: Tapera, B. Pickel, 12-XII-1934 (SP). Rio de Janeiro: km 5 da estrada Rio-Santos, W. Hoehne 5898, 3-XI-1964 (SP). São Paulo: Cananéia, Ilha do Bom Abrigo, M. Kirizawa 1997, 9-XII-1987 (SP); Ilha do Cardoso, F. Barros 478, 8-X-1980 (SP), F. Barros 718, 1-VI-1982 (SP), A. Custodio Filho 500, 12-XII-1980 (SP), D. A. De Grande \& E. A. Lopes 189, 13-XII-1978 (SP); H. F. Leitão Filho \& J. Y. Tamashiro 17998, 3-XII-1985 (UEC); H. F. Leitão Filho et al. 10825,1 1-XII-1979 (UEC); M. C. H. Mamede \& R. Andreata 101, 18-X-1988 (SP); M.C.H. Mamede et al. 189, 25-X1988, R.D. Marassi et al. 65, 24-XI-1982(SP); O. Yano, 9-XI-1977; Caraguatatuba, F.C. Hoehne \& A. Gehrt, 8-XII-1939 (SP); Ipanema, O. Handro, 28-XII-1940 (SP); Itanhaem, A. Loefgren (Comissão Geográfica e Geológica de São Paulo n. ${ }^{0}$ 1576); 23X-1891 (SP); Praia Grande, Mongaguá, A.S. Grotta \& J. Bartolomeu,29-XI-1953 (SPF); São Sebastião, Bertioga, P.E. Gibbs et al.3533, 10-XI- 1976 (SP,UEC); Ubatuba, 2 Km do rio Acaraú, J. Fontella \& C. Moura 73, 4-XI-1961 (SP). Santa Catarina: Florianópolis, F.Bertagnolli Junior, 26-IV-1939 (SP). Sem localidade: cultivada na fazenda Riqueza, Campinas, São Paulo, A.P. Viegas et al., 6-XI-1938 (SP); cultivada em Petrópolis, Rio de Janeiro, C. Spannagel (SP). 


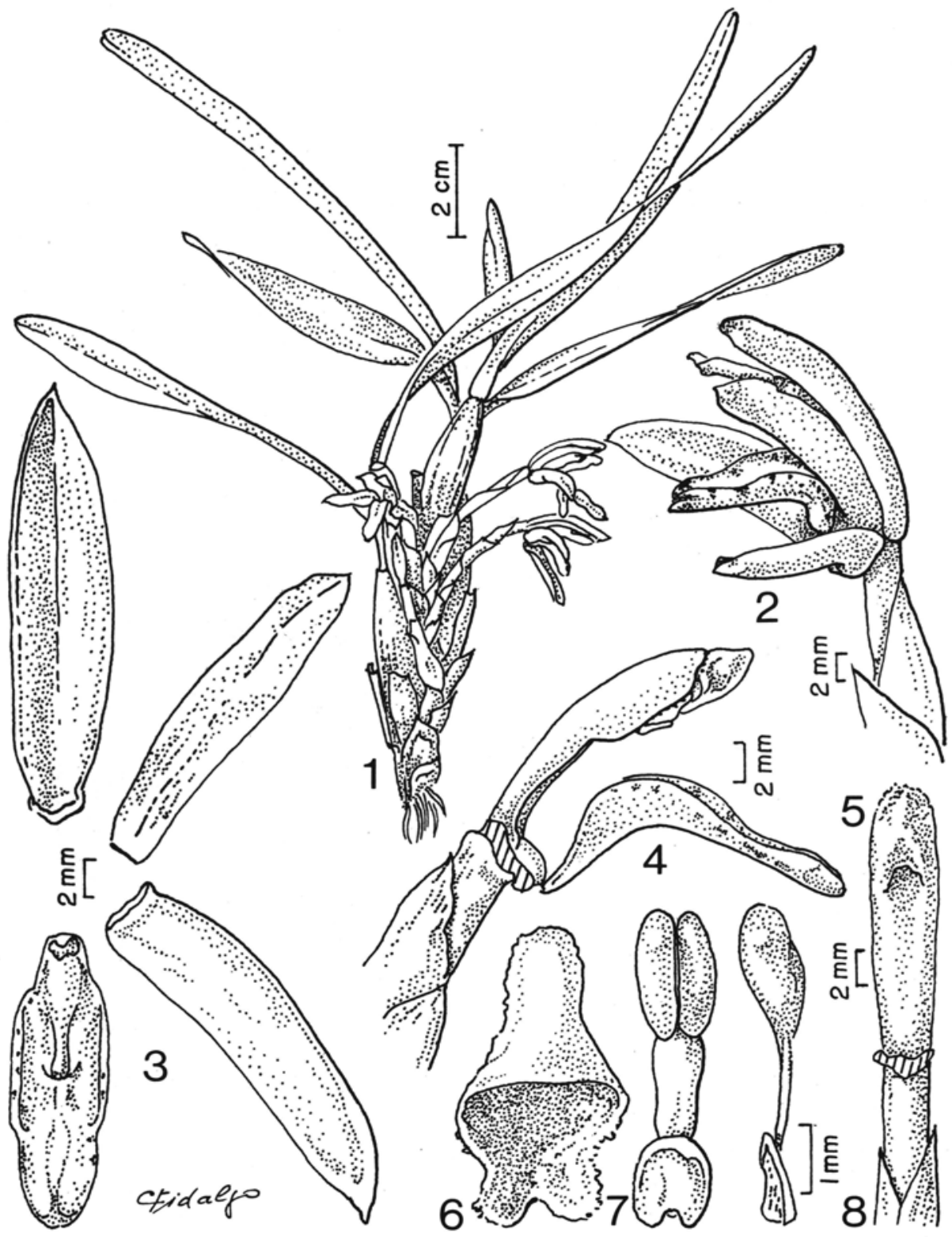

Figuras 1-8. Maxillaria friedrichsthalii Rchb. f. 1. aspecto geral. 2. flor vista lateralmente. 3. flor dissecada. 4. coluna e labelo vistos lateralmente. 5. coluna vista ventralmente (antera retirada). 6. anteras. 7. polinário visto dorsalmente e lateralmente. 8. coluna vista ventralmente (antera retirada). 


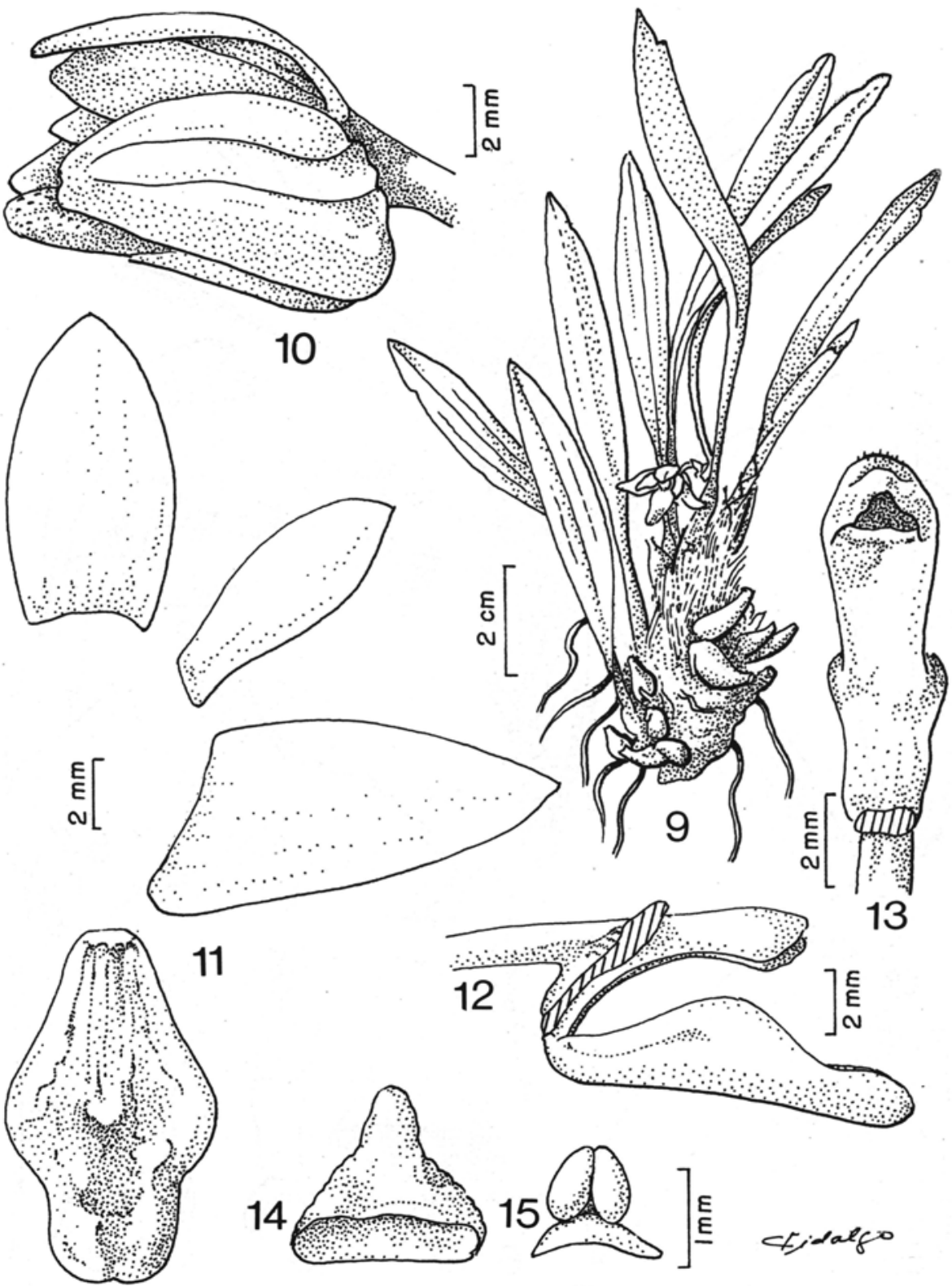

Figuras 9-15. Maxillaria perpava Garay \& Dunsterv. 9. aspecto geral. 10. flor vista lateralmente. 11. flor dissecada. 12. colunae labelo vistos lateralmente. 13. coluna vista ventralmente (antera retirada). 14. antera. 15. polinário. 
Maxiliaria friedrichsthalii Rchb. f., Bot. Zeit. 10:858. 1852.

M. aciantha Rchb. f., Bot. Zeit. 10:858. 1852.

M. scorpioidea Krzl., Kungl. Sv. Vetensk. Akad. Handl. 46(10):71. 1911.

Camaridium scorpoideum (Krzl.) Hoehne, Arq. Bot. Est. S. Paulo 2(4) :72. 1947.

Figs. 1-8.

Esta espécie que, segundo Ames \& Correll (1952), é comum desde o México até o Panamá, foi coletada rara e ocasionalmente no Brasil. É interessante notar que até o momento ela não foi encontrada nas regiões intermediárias entre o Brasil e a América Central. Dentre as congêneres brasileiras distingue-se bem pelo seguinte conjunto de caracterítiscas: rizoma longo, flores emergindo das axilas das bainha do rizoma, flores muito carnosas, labelo simples e estreito, com superfície pegajosa, e polinários com estipe longo e estreito e viscídio estreito.

Material estudado: Brasil: Rondônia: Rio Ji-Paraná, barragem da U.H.E. do JiParaná, P. Martuscelli, fl. cult. 2-X-1991 (SP).

Maxillaria perpava Garay \& Dunsterv., Venez. Orch. Ill. 6:37. 1976.

Basiônimo: Bifrenaria minuta Garay, Bot. Mus. Leafl. Harv. Univ. 15:206. 1958. (non Maxiliaria minuta Cogn.)

Figs. 9-15.

Esta espécie era conhecida, até o momento, apenas da Venezuela. Sua ocorrência é constatada, agora, no Brasil.

Material estudado: Brasil: Amazonas: Presidente Figueiredo, região da Usina Hidroelétrica de Balbina, J.B.F. da Silva, fl. cult. 14-XI-1989 (SP).

Plastystele ovalifolia (Focke) Garay \& Dunsterv., Venez. Orch. Ill. 2:268. 1961.

Basiônimo: Stelis ovalifolia Focke, Tijdschr. Natuur. Wetensch. 2:202. 1849.

Pleurothallis ovalifolia (Focke) Rchb. f., Walp. Ann. Bot. Syst. 6:188. 1861.

Pleurothallis rhomboglossa Rchb. f., Flora 48:276. 1865.

Figs. 16-22.

Esta espécie é conhecida, até o momento, das Indias Ocidentais, Trinidad, Venezuela, Guiana, Suriname e, mais recentemente, Brasil. Caracteriza-se, dentre as demais espécies brasileiras do gênero, por seu hábito reptante, ramicaule muito curto, folhas obovais a arrendondadas e inflorescências mais altas que as folhas.

Material estudado: Brasil: Pará: Castanhal, Igarapé Apéu, F. Barros, 28-I-1988 (SP); Mosqueiro, J.M. Pires 12162, 22-V-1971 (IAN, SP); São Felix do Xingu, J.B.F. da Silva, 26-II-1988 (SP).

Pseudolaelia canaanensis (Ruschi) F. Barros comb. nov.

Basiônimo: Renata canaanensis Ruschi, Publ. Arq. Publ. Est. Ep. Santo 3:6. 1946.

Embora Ruschi (1946), ao estabelecer o gênero Renata, não tenha ressaltado sua afinidade com Pseudolaelia Porto \& Brade, essa afinidade é incontestável, tanto que Brieger (1977), Pabst \& Dungs (1977) e Ruschi (1986), utilizaram caracteres de 


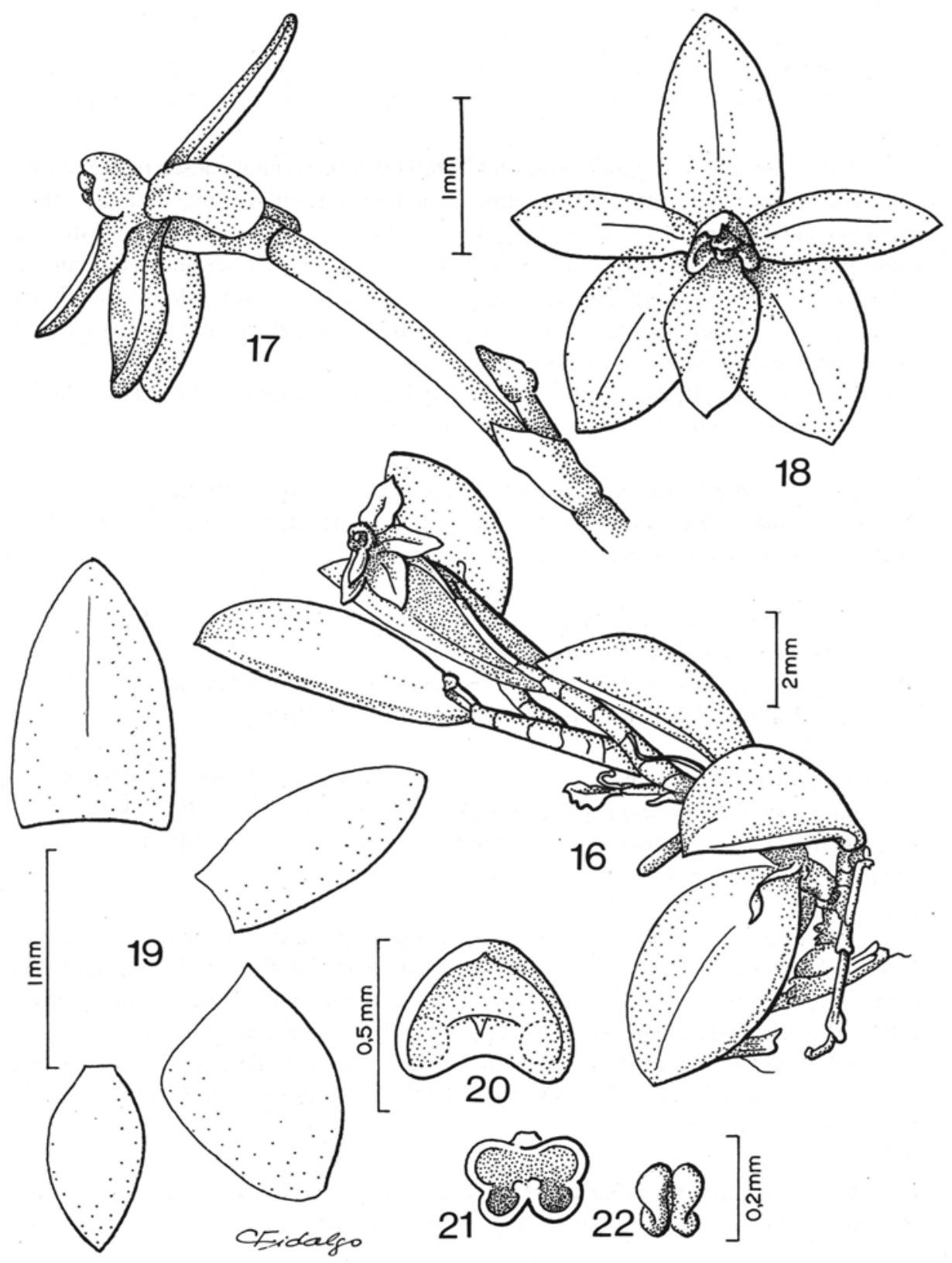

Figuras 16-22. Platystele ovalifolia (Focke) Garay \& Dunsterv. 16. aspecto geral. 17. flor vista lateralmente. 18. flor vista frontalmente. 19. flor dissecada. 20. coluna vista do ápice. 21. antera. 22. polínias. 
valor bastante discutível, na diferenciação entre esses dois gêneros, como: cor das flores, números de folhas apicais do pseudobulbo e presença ou ausência de lobos laterais de labelo. Algumas espécies de Pseudolaelia descritas mais recentemente, têm se mostrado intermediárias com Renata, em algumas de suas características. As únicas diferenças que persistem são o número maior de folhas no ápice do pseudobulbo de Renata e a inflorescência, que é um racemo em Pseudolaelia e uma panícula em Renata; ambas não justificam a manutenção de um gênero. Ressalte-se que a estrutura floral é basicamente a mesma nos dois gêneros e a estrutura da coluna é idêntica.

Material estudado: Brasil: Espírito Santo: Santa Tereza, Estação Biológica do Museu Nacional, H. Sick, 7-III-1961 (HB); Santa Tereza, Vale de Canaan, A. Ruschi, 23-III1946 (HB); Morros do Vale de Canaan, R.A. Kautsky 426, 6-IV-1974 (HB); cultivada no Jardim Botânico de São Paulo, A. Ruschi, 25-III-1949 (HB,SP). Sem localidade, H.D. Bicalho, 26-III-1969 (SP).

\section{Agradecimentos}

A Carmen S.Z. Fidalgo pela execução das figuras e a Rosana Paiva Peres pela datilografia dos originais.

\section{Referências bibliográficas}

Ames, O. \& Correl, D.S. 1952. Orchids of Guatemala. Fieldiana, Bot. 26 (1): 1-727.

Brieger, F.G. 1977. Die Orchideen. In: Brieger, F.G.; Maatsch, R. \& Senghas, K. (eds.) 3(9). Berlin: Paul Parey.

Cogniaux, A. 1898. Orchidaceae. In: Martius, C.F.P.; Eichler, A.G. \& Urban, I. (eds.) Flora Brasiliensis 3(5): 1-664.

Garay, L.A. \& Sweet, H.R. 1974. Orchidaceae. In: Howard, R.A. (ed.). Flora of Lesser Antilles. Arnold Arboretum.

Hoehne, F.C. 1942. Orchidaceae. In: Hoehne, F.C. (ed.) Flora Brasilica 12(6):1-218.

Hoehne, F.C. 1952. As orchidáceas referidas e desenhadas para a "Flora Flum." de Frei José Mariano da Conceição Vellozo e sua atual nomenclatura. Arch. Bot. S. Paulo 2(6): 141-146.

Pabst, G.F.J. \& Dungs, F. 1975. Orchidaceae Brasilienses I. Hildesheim: Kurt Schmersow.

Pabst, G.F.J. \& Dungs, F. 1977. Orchidaceae Brasilienses II. Hildesheim: Kurt Schmersow.

Ruschi, A. 1946. Orquidáceas novas do Estado do Espírito Santo. Publ. Arq. Publ. Est. Esp. Santo 3:1-56.

Ruschi, A. 1986. Orquídeas de Estado do Espírito Santo. Rio de Janeiro: Expressão e Cultura.

Vellozo, J.M.C. 1827. Flora Fluminensis Icones 9. Paris: Senefelder.

Vellozo, J.M.C. 1881. Flora Fluminensis. Arch. Mus. Nac.,Rio de J. 5:1-461. 